\title{
Zwischen Firmenbewusstsein und Wachstumskritik. Empirische Befunde aus einem Industriebetrieb
}

Die seit 2008 virulente Finanzmarktkrise und ihre gravierenden gesellschaftlichen Folgen haben auch mit Blick auf die Legitimität des Kapitalismus als Gesellschaftsform ihre Spuren hinterlassen. In diesem Zusammenhang sind die Gesellschaftsbilder von Lohnabhängigen wieder zu einem Thema geworden. Auf der Grundlage von Untersuchungen in einem Industriebetrieb zeigt der Beitrag, dass sich hinter dem Unbehagen am Finanzmarktkapitalismus auch eine ökologisch inspirierte Wachstumskritik verbirgt.

KLAUS DÖRRE, HAJO HOLST, INGO MATUSCHEK

\section{Einleitung}

Einst ein wichtiges Feld arbeitssoziologischer Forschungen, war die wissenschaftliche Diskussion um das Bewusstsein von Lohnabhängigen lange Zeit verstummt. Studien, die wie ein Klassiker dieses Genres - das „Gesellschaftsbild des Arbeiters" (Popitz et al. 1957) thematisierten, schienen nicht mehr zeitgemäß. Unter dem Eindruck einer verbreiteten Alltagskritik am Kapitalismus, die auch in demoskopischen Befunden zum Ausdruck kommt (Institut für Demoskopie 2012), kehrt das Interesse an solchen Forschungen zurück. Empirische Untersuchungen befassen sich mit dem Ungerechtigkeitsbewusstsein von Lohnabhängigen (Dubet 2008) und dem Zerfall politischer Orientierungen von Arbeitern (Beaud/Pialoux 2004), sie thematisieren die Gesellschaftsbilder eines neuen Dienstleistungsproletariats (Bahl/Staab 2010) oder befassen sich mit dem Krisenbewusstsein gewerkschaftlich aktiver Gruppen (Detje et al. 2011). Bei aller Unterschiedlichkeit belegen diese Studien, dass die Akzep$\tan z$ des Kapitalismus eher dem Empfinden von Alternativlosigkeit als einer auf Überzeugung beruhenden Zustimmung zu diesem Gesellschaftssystem geschuldet ist.

Doch was genau verbirgt sich hinter den kritischen Gesellschaftsbildern von abhängig Beschäftigten? Unsere These lautet, dass es sich beim alltäglichen Unbehagen am zeitgenössischen Finanzmarkt-Kapitalismus keineswegs um eine bloße Wiederkehr altbekannter Muster der Sozial- und
Gesellschaftskritik handelt. Die Zustimmung zur kapitalistischen Wirtschaft und ihren Institutionen bröckelt selbst in noch relativ geschützten Stammbelegschaften, weil sich Arbeiter, Angestellte und auch Führungskräfte in einer Tretmühle wähnen, aus der es kein Entrinnen gibt. Hinter dem Unbehagen am Kapitalismus verbirgt sich vielfach eine lebensweltlich begründete Kritik an einem alles vereinnahmenden Wettbewerbs- und Wachstumsimperativ. Zur Begründung dieser These skizzieren wir zunächst Fragestellung und empirische Basis unserer Untersuchung (Abschnitt 2), beschreiben sodann die eigentümliche Kombination aus Firmenbewusstsein (3) und unterschiedlichen Typen von Kapitalismuskritik (4 und 5), um schließlich die Frage nach dem Zusammenhang von Arbeitsbewusstsein und Wachstumskritik wieder aufzugreifen (6) und arbeitspolitische Implikationen zu beleuchten (7).

\section{Untersuchungsziele und empirische Basis}

Dass sich in der Belegschaft eines exportorientierten Fahrzeugherstellers wachstumskritische Orientierungen finden, ist eine empirische Fundsache, die wir so nicht erwartet hatten. Die nachfolgenden Ausführungen beruhen auf empirischen Studien, die wir zwischen 2009 und 2012 in 
einem Großbetrieb der Fahrzeugbranche durchführen konnten. Im Zentrum der Untersuchung stand die Wahrnehmung und Bewertung betrieblicher Flexibilisierungsinstrumente durch Beschäftigte und Führungskräfte. Von den Ergebnissen einer Belegschaftsbefragung in ostdeutschen Betrieben eines Optoelektronik-Unternehmens angeregt (Behr et al. 2013, S. 54ff.), wollten wir zudem die Relevanz eines Bewusstseinsmusters prüfen, das wir in der Formel "guter Betrieb, schlechte Gesellschaft" zusammengefasst hatten (Dörre et al. 2011). Ursprünglich waren wir davon ausgegangen, dass die Kombination aus hoher Identifikation mit der Firma und teilweise harscher Kapitalismuskritik der Besonderheit eines Stiftungsunternehmens geschuldet war. In weiteren Befragungen hatte sich jedoch bereits angedeutet, dass es sich bei den rekonstruierten Gesellschaftsbildern keineswegs um das Sonderbewusstsein einer einzelnen Belegschaft handelte. Im Kontext der globalen Wirtschaftskrise von 2008/09 sollte daher untersucht werden, $o b$ in einem westdeutschen Industriebetrieb ähnliche Orientierungen zu finden sind.

In diesem Zusammenhang formulierten wir einige untersuchungsleitende Thesen (Dörre/Matuschek 2013, S. 41ff.), von denen mit Blick auf alltägliche Wachstumskritik vor allem drei von Bedeutung sind. Die erste These behauptete eine hohe Identifikation von Stammbelegschaften mit Betrieb und Unternehmen („guter Betrieb“), die zweite These ging von einer starken Verbreitung von gesellschafts- und kapitalismuskritischer Einstellungen in den Belegschaften aus („schlechte Gesellschaft"), während die dritte These eine Erwartung formulierte, der zufolge kapitalismuskritische Orientierungen höchst unterschiedliche Handlungsstrategien nahelegen können („fragmentiertes Lohnabhängigenbewusstsein"). Wachstumskritik war zunächst nicht explizit unser Untersuchungsgegenstand. Das änderte sich im Verlauf der Untersuchung. Den genannten Thesen gingen wir in mehreren quantitativen und qualitativen Erhebungen nach. 2009 starteten wir mit einer problemgenerierenden Befragung von 54 Beschäftigten (plus sechs aktualisierende Experteninterviews 2011) aller Hierarchiestufen. 2012 folgten 35 problemzentrierte Interviews. Zuvor (2010) konnten wir Arbeiter und produktionsnahe Angestellte im Rahmen einer standardisierten Erhebung untersuchen $(\mathrm{n}=1.442)$. Der Fragebogen umfasste die Themenblöcke Flexibilisierung der Arbeit, Krisenerfahrungen, $\mathrm{Zu}$ kunftsperspektiven, Work-Life-Balance, wirtschaftliche Entwicklung, Betriebsrat und Gewerkschaften sowie Arbeitsund Gesellschaftsbewusstsein. Um Facetten von Gesellschaftsbildern zu erfassen, wurden Fragebatterien aus den Belegschaftsbefragungen in ostdeutschen Betrieben so weit wie möglich repliziert, thematisch orientierten sich die Fragebatterien an neueren Untersuchungen zu politischen Milieus (Neugebauer 2007). Die abgefragten Themen (Verteilungsgerechtigkeit, Wahrnehmung sozialer Ungleichheit, Haltung zur kapitalistischen Wirtschaftsweise) sind zudem als Rückgriff auf die klassische Arbeiterbewusstseinsforschung (Dörre/Matuschek 2013, S. 39ff.) zu verstehen.
Unsere Untersuchung beschränkte sich nicht auf Produktionsarbeiter und produktionsnahe Angestellte. 2011 wurden Sachbearbeiter und Führungskräfte des Werks in unsere Analysen einbezogen; der Fragebogen war in weiten Teilen mit dem der Belegschaftsbefragung identisch (Sachbearbeiter $n=618$, Führungskräfte $n=262$ ). Die multivariat angelegte Auswertung erfolgte mit PASW/SPSS. Die standardisierten Datensätze wurden sowohl einzeln ausgewertet als auch zusammengeführt in die Analyse einbezogen. Auf der Grundlage einer Clusteranalyse konnten wir vier typische Alltagsphilosophien bzw. Kritikmuster identifizieren, deren Kohärenz wir Ende 2012 in einer kleinen qualitativen Erhebung nachgingen. Es waren nur sechs Tiefen-Interviews nötig, um die zuvor identifizierten Bewusstseinstypen qualitativ abzubilden. In diesen Interviews wurde systematisch nach der Wahrnehmung ökologischer Krisen und der Beurteilung wachstumskritischer Argumente gefragt. Die im Beitrag zitierten Passagen sind diesen Interviews entnommen.

Unsere Befunde gelten für die Beschäftigten eines Werks, dessen Belegschaft sich in der Produktion zu 6\% aus Frauen und zu $94 \%$ aus Männern zusammensetzt. Auf der Sachbearbeiterebene finden sich immerhin $21 \%$ weibliche Beschäftigte, während in der Gruppe der Führungskräfte nur jede zwanzigste Angestelltenposition von einer Frau besetzt ist. Insgesamt haben wir es mit einer vergleichsweise hoch qualifizierten Belegschaft zu tun, in der männliche Facharbeiter im Alter zwischen 35 und 54 Jahren die zahlenmäßig dominierende Gruppe stellen. Ein Großteil der Belegschaft rekrutiert sich aus der Region, und nicht wenige Beschäftigte sind bereits 30 Jahre und länger im Werk angestellt. Insofern haben wir es, wie schon bei den Untersuchungen in der ostdeutschen Optoelektronik, mit Sonderbedingungen zu tun. Da wir in höchst unterschiedlichen betrieblichen Konstellationen auf sehr ähnliche Bewusstseinsmuster gestoßen sind, dürften unsere Befunde jedoch über einzelne Belegschaften hinaus von Bedeutung sein. ${ }^{1}$

\section{Identifikation mit der „kleinen Welt“ des Betriebs}

Was hat es nun mit der Kapitalismus- und Wachstumskritik von Arbeitern und Angestellten in der Automobilindustrie auf sich? Unsere ersten empirischen Befunde schienen zunächst etwas völlig anderes zu signalisieren als Kritik am Wachstumskapitalismus. Charakteristisch für die von uns untersuchte Belegschaft ist, wie in der ersten These unterstellt, zunächst ihre hohe Identifikation mit dem Werk (das wir

1 Zu den hier verwendeten und zu weiteren Datensätzen vgl. Dörre et al. 2013, S. $277 f f$. 
im Folgenden mit dem Buchstaben $\mathrm{Z}$ bezeichnen), dem Unternehmen und, was hier zusätzlich hinzukommt, der Region. Fast $60 \%$ der befragten Arbeiter und produktionsnahen Angestellten bezeichnen das Unternehmen gegenüber Freunden und Bekannten als besonders guten Arbeitgeber, ein weiteres Drittel macht das immerhin gelegentlich. Mit „Haut und Haar“ dem regionalen Betrieb zugehörig fühlen sich mehr als $58 \%$ der Befragten. Für fast $29 \%$ trifft dies teilweise zu und nur eine Minderheit von $13 \%$ entwickelt eine solche Identifikation gar nicht. Bei der Identifikation mit der „kleinen“ Betriebswelt handelt es sich aber keineswegs um eine monistische Bewusstseinsform, die Unterschiede zwischen Managementhierarchie und Beschäftigten verwischen würde. Das Gegenteil kann der Fall sein. Die sicherlich unterschiedlich ausgeprägte Positividentifikation kann auch zur Quelle für vehemente Kritik an der Arbeitssituation, an dem betrieblichen oder dem Unternehmensmanagement werden. Die Identität als „Z'ler“ gründet sich auf gewachsene Bindungen an Werk und Region ebenso wie auf ein bestimmtes Produkt. Dann erst folgt das Unternehmen - auch hier sind es das Produkt und eine gewisse Egalität (nämlich das gleiche Automobil zu fahren wie der Chef), die Identifikation erzeugen. Ein befragter Anlagenbetreuer beschreibt sein Selbstverständnis mit folgenden Worten:

„Wir sind Z’ler [...] Also [...] vor 25 Jahren, hab ich immer gesagt, ich bin stolz! Jetzt, es hat sich einiges gewandelt. Man identifiziert sich auch nimmer so, aber ich bin schon noch ein Z'ler, und [...] ich bin froh oder stolz drauf, auch hier arbeiten zu dürfen. Sagen wir mal, also das ist schon ein Privileg, weil, wenn man bedenkt, was es für andere Möglichkeiten [gibt, d. A.]. Oder man kennt ja auch Kolleginnen und Kollegen, die in anderen Firmen arbeiten [...], da geht's ja noch heftiger zu. Ich denk mal, es liegt nicht in meiner Hand, über den Vorstand zu entscheiden oder über die Philosophie von meinem Vorstandsvorsitzenden, weil das macht [...] viele unzufrieden. Der Stress hat schon zugenommen. Früher waren ja die Unternehmen so eingestellt, also [...], so wie ein Kleinunternehmer. Da ist halt der Chef [...], dem liegt das Herz dran. Heutzutage sind's eigentlich nur noch irgendwelche Aktienindexe, Aktieninvestoren wollen Geld, wollen ein bestimmtes Ergebnis haben, wollen 'ne bestimmte Ausschüttung haben und der Vorstand ist da bemüht, denen gerecht zu werden, und es interessiert auch nicht, wie das erreicht wird [...], nicht nur die Firma hat sich geändert, sondern auch unsere ganze Lebensweise hat sich ja auch in diesen drei Jahrzehnten geändert. Wir sind ja da viel schnelllebiger geworden und viel,will ich haben' und wie kann ich, nur noch Ich-betrachtet das anschauen und nicht mehr zu gucken, Mensch, wie können WIR's zusammen machen, sondern ich, Chef, ich brauche mehr Geld und der andere, ist mir egal, was der kriegt. ICH will mehr“ (Interview).

Im Vergleich zum regionalen Umfeld, dem Lohnniveau in kleineren Betrieben und der Realität prekärer Beschäftigung erscheinen Werk und Unternehmen trotz aller Kritik an der Shareholder-Value-Orientierung der Konzernleitung geradezu als Refugium sozialer Sicherheit. Wer „beim Z.“ arbeitet und zur Stammbelegschaft gehört, der weiß, dass seine Beschäftigung einigermaßen sicher ist. Zwar nimmt der Stress im Arbeitsprozess zu, und gemessen an der, sicher positiv überhöhten, Vergangenheit stört das herzlose, finanzkapitalistische Management des Konzerns. Die positive Identifikation, das Selbstverständnis als „stolzer Z’ler“, wird dennoch oder gerade deshalb aufrechterhalten.

Dies ist möglich, weil Kritik, die sich auf die kleine Welt des Werks und die schon erheblich größere des Konzerns bezieht, subjektiv durch zwei Beobachtungen relativiert wird. Erstens sind nicht nur das Unternehmen und seine Manager „egoistischer“ geworden, sondern die gesamte Gesellschaft hat sich in diese unerwünschte Richtung bewegt. Im Konzern spiegelt sich somit nur eine allgemeine Veränderung der Lebensweise und der Kultur, die das „Ich will mehr!" in den Mittelpunkt stellt. Zweitens ist das Werk, gemessen an anderen Betrieben, ein „ausbeutungsfreier“ Ort. Ausbeutung wird in diesem Fall als unfairer Tausch definiert, der sich von Arbeitsverhältnissen und Tauschbeziehungen abgrenzen lässt, auf welche die Beschäftigten über ihre Betriebsräte und die Gewerkschaften Einfluss nehmen können. Ausbeutung steht somit subjektiv für Übervorteilung, das Erleben ,ungerechter Ungleichheiten“, für eine radikale Negation von Leistung (Dubet 2008, S. 119). Das Fehlen solcher Ausbeutungsformen macht die Beschäftigung im Werk subjektiv zu einem Privileg. Und diese relativ privilegierte Situation, von der man weiß, dass sie schon für jüngere Beschäftigte so nicht mehr gilt, möchte man - auch durch große Leistungs- und Flexibilitätsbereitschaft - verteidigen. Insofern handelt es sich bei der Positividentifikation mit dem Werk und - abgeschwächter - dem Unternehmen nicht um „falsches Bewusstsein“. Vielmehr bezeichnet die Zugehörigkeit zur Stammbelegschaft einen sozialen Ort, dem man sich zugehörig fühlt, weil er Teilhabe am gesellschaftlichen Leben ermöglicht.

\section{Kritik am Finanzmarkt-Kapitalismus}

Trotz der hohen Identifikation mit Werk und Unternehmen sind, wie in unserer zweiten These vermutet, kapitalismuskritische Einstellungen in allen Statusgruppen der untersuchten Belegschaft weit verbreitet. Bei der Erfassung dieser Einstellungen haben wir uns an der klassischen Arbeiterbewusstseinsforschung orientiert. Popitz et al. (1957, S. 237ff.; vgl. auch Dörre/Matuschek 2013, S. 36ff.) hatten seinerzeit ein dichotomisches Gesellschaftsbild als übergreifende Gemeinsamkeit eines Kollektivbewusstseins von Industriearbeitern ausgemacht. Dieser Befund wurde von der späteren Arbeitsbewusstseinsforschung relativiert und differenziert (vgl. Schumann 2013); er ist, wie unsere Daten belegen, in gewisser Weise aber noch immer relevant. Wir haben 
die Alltagskritik am Gegenwartskapitalismus in drei Dimensionen abgefragt:

(1) Dichotomie: Eine große Mehrheit der befragten produktionsnahen Arbeiter und Angestellten geht davon aus, in einer Gesellschaft zu leben, die sich sozial zunehmend polarisiert. Die Aussage, dass es in der Gesellschaft nur noch ein Oben und ein Unten gibt, halten $67 \%$ der Befragten des Automobilherstellers für vollständig oder eher richtig.

(2) Verteilungsgerechtigkeit: Dass der gesellschaftliche Reichtum viel gerechter verteilt werden könnte, ist fast schon ein Kollektiv(vor)urteil: $74 \%$ der produktionsnahen Arbeiter und Angestellten stimmen dieser Aussage vollständig oder eher zu.

(3) Wirtschaftssystem: Aufgrund der wahrgenommenen Spaltungen und Ungerechtigkeiten bröckelt die Legitimationsgrundlage der kapitalistischen Wirtschaft. Die Aussage „Das heutige Wirtschaftssystem ist auf Dauer nicht überlebensfähig“ hält eine Mehrheit der Arbeiter und produktionsnahen Beschäftigten für zutreffend ( $54 \%$ stimmen voll oder eher zu). Auffällig ist der hohe Anteil an Befragten, die unentschieden sind (34\%; die Werte beziehen sich auf die separate Auswertung der Datensätze).

Auf der Basis einer Clusteranalyse lassen sich im untersuchten Automobilwerk vier typische Bewusstseinsmuster unterscheiden, die wiederum zwei Hauptclustern zugeordnet werden können. Wir bezeichnen diese Hauptcluster als die Kritiker (ca. $42 \%$ ) und die Moderaten (gut 58\%). Die Kritiker formulieren auf der Basis eines ausgeprägt dichotomischen Gesellschaftsbildes eine scharfe Kritik am Gegenwartskapitalismus, die insbesondere Machtungleichgewichte und Verteilungsungerechtigkeiten thematisiert. Die Moderaten changieren als Gruppe zwischen einer - allerdings weniger klar artikulierten - Kritik und einem eher affirmativen Gesellschaftsbild. Auffällig ist, dass wir kein Cluster identifizieren konnten, dessen Mitglieder das Wettbewerbsregime des Gegenwartskapitalismus aktiv bejahen. Die Kritiker teilen sich in Systemkritiker und Wettbewerbsindividualisten auf. Während die Systemkritiker im Einklang mit ihrer Gesellschaftskritik ein inklusives Gesellschaftsverständnis entwickeln, artikulieren die Wettbewerbsindividualisten ein Distinktionsbedürfnis, das erhebliche Ausgrenzungspotenziale $^{2}$ in sich trägt. Das Hauptcluster der Moderaten wiederum teilt sich in Wettbewerbskorporatisten und Affirmative. Die Wettbewerbskorporatisten ähneln in einigen Aspekten den Wettbewerbsindividualisten. Allerdings scheint das Ausgrenzungspotenzial, das bei den Wettbewerbsindividualisten trotz schärferer Kapitalismuskritik zum Tragen kommt, bei den Wettbewerbskorporatisten weniger ausgeprägt zu sein. Auch die Befragten, die dem Cluster der Affirmativen zuzurechnen sind, zeigen sich situativ durchaus kritisch; insgesamt fällt ihr Gesellschaftsbild jedoch deutlich positiver und ihre Kritik weniger grundsätzlich aus.
Wenn überhaupt, so findet sich eine Verbindung zwischen betrieblichem Arbeitsbewusstsein und Gesellschaftsbild bei den Systemkritikern. Kapitalismuskritik verbindet sich bei diesen Befragten mit einer ebenso kritischen Sicht der Arbeitsbedingungen im Untersuchungsbetrieb. Nicht einmal $2 \%$ dieser Befragten lehnen das Statement ab, dass die Belastungen in den letzten Jahren deutlich angestiegen sind. Zeitdruck gehört zum Alltag, ebenso das Gefühl, am Abend erschöpft zu sein. Systemkritiker halten die Spielregeln des Systems grundsätzlich für veränderbar; das auch, weil sie auf eine funktionierende Mitbestimmung und wirkungsmächtige Gewerkschaften setzen. Ihr kritisches Gesellschaftsbild motiviert sie daher tendenziell zu kollektivem Engagement. Kritik üben sie sowohl an den Auswüchsen des Finanzmarkt-Kapitalismus als auch am Kapitalismus als Gesellschaftssystem.

Anders ist das bei den Wettbewerbsindividualisten. Sie betrachten die Gesellschaft als starres System und haben weniger Zutrauen zu Mitbestimmung und Gewerkschaften. Daher setzen sie vor allem auf sich selbst. Ihr Individualismus gründet sich aber nicht auf Aufstiegshoffnungen. Das Abwägen individueller Möglichkeiten zielt auf Statuserhalt. Es gibt etwas zu verteidigen, und die einzige und entscheidende Ressource ist die individuelle Leistungs- und Flexibilitätsbereitschaft. Der Kapitalismus erscheint als starres Wettkampfsystem, dessen permanente Bewährungsproben individuell bewältigt werden müssen. Dementsprechend setzt man nicht auf kollektives Engagement - und wenn Solidarität praktiziert wird, dann exklusiv und kompetitiv, also mit ausgeprägter Abgrenzung vor allem nach unten. Der deutliche Kontrast innerhalb des Kritiker-Clusters belegt, dass Kapitalismus- und Gesellschaftskritik, wie in der dritten These erwartet, höchst unterschiedliche Verhaltensweisen und Handlungsstrategien nahelegen können. Je hermetischer das kapitalistische System subjektiv konstruiert wird, desto eher legt Kapitalismuskritik ein individualistisches Verhalten nahe, das im Resultat jenen Käfig, in welchem man sich gefangen sieht, umso geschlossener erscheinen lässt.

Bemerkenswert ist in diesem Zusammenhang eine weitere Erkenntnis. Generell hält man Gewerkschaften für nötig, teilweise auch für mächtig und durchsetzungsstark. Eine umfassende Gesellschaftsveränderung ist jedoch nichts, was eine zu über $90 \%$ organisierte Belegschaft ihrer Organisa-

2 Wir haben diese Bewusstseinsform, die sich nicht nur gegen "oben", sondern auch von "unten" und „anders" abgrenzt, an anderer Stelle als exklusive Solidarität bezeichnet. Empirisch kann exklusive Solidarität anhand der Einstellung zu prekär Beschäftigten und Erwerbslosen identifiziert werden. Trotz mehrheitlicher Ablehnung von Hartz IV sind $54 \%$ der Arbeiter und produktionsnahen Angestellten der Ansicht, es müsse mehr Druck auf Langzeitarbeitslose ausgeübt werden; $51 \%$ meinen, eine Gesellschaft, in der man jeden auffange, sei auf Dauer nicht überlebensfähig (vgl. Dörre 2014). 
tion zutrauen würde. Es gibt keine oder allenfalls sehr schwach ausgeprägte kognitive Schemata, mit deren Hilfe sich kritische Gesellschaftsbilder synchronisieren und aktivieren ließen. Die Gewerkschaft wird „bei diesen gesellschaftspolitischen Themen [...] nicht unbedingt ernst genommen", bringt ein Angestellter dieverbreitete Grundhaltung auf den Punkt. Auch weil sie sich im organisierten politischen Spektrum nicht zu verorten vermag, bleibt die verbreitete Kapitalismuskritik weitgehend orientierungslos (ähnlich Detje et al. 2011).

Für die relative Stabilität der Bewusstseinsmuster spricht, dass sie sich trotz sozialer Heterogenität in allen Statusgruppen finden (Abbildung 1). Die Gruppe der Affirmativen ist, was wenig überrascht, bei den Führungskräften am größten, doch selbst hier machen die Systemkritiker immerhin 13\% aus. Interessant ist, dass die Systemkritiker bei den Sachbearbeitern quantitativ in etwa so bedeutend sind wie bei den produktionsnahen Befragten. Hingegen ist der Typus der Wettbewerbsindividualisten primär bei den Arbeitern und produktionsnahen Angestellten verbreitet.

\section{Kapitalismuskritik und fragmentiertes Bewusstsein}

Gemeinsam mit je eigenen Arbeitserfahrungen trägt dies zu einer Ausdifferenzierung des Gesellschaftsbewusstseins von Lohnabhängigen bei. Die Überzeugung, dass soziale Spaltungen sich vertiefen, findet sich allerdings nicht allein bei den produktionsnah Beschäftigten, sondern auch bei Sachbearbeitern und Führungskräften. $62 \%$ der Sachbearbeiter und $47 \%$ der Führungskräfte sind der Ansicht, der Wohlstand könne gerechter verteilt werden. Doch während sich das Ungerechtigkeitsbewusstsein offenbar über die Statusgruppen hinweg verallgemeinert, verliert es jene kohäsive Kraft, die das dichotomische Bewusstsein einer Schicksalsgemeinschaft von Arbeitern einst erzeugt hatte (Popitz et al. 1957).

Die Systemkritiker halten zwar einen Systemzusammenbruch aufgrund von externen Schocks (ökologische Krise) und inneren Verwerfungen (ökonomische Krise) für denkbar, teilweise gar für wahrscheinlich. Die Erschütterungen des kapitalistischen Systems werden aber nicht als Verheißung einer besseren Gesellschaft, sondern eher als Verlust, als Bedrohung des Erreichten interpretiert. Dieser konservierende Grundzug verbindet radikale und moderate Kapitalismuskritiker. Über alle Typen hinweg hat die alltägliche Kapitalismus- und Gesellschaftskritik etwas Defensives. Anpassung an systemische Zwänge bedeutet allerdings - hier müssen wir frühere Annahmen korrigieren - nicht automatisch, die Ellenbogen auszufahren, um sich im täglichen Überlebenskampf als der Stärkere zu erweisen. Ein durchaus realistisches Kalkül kann auch darin bestehen, den kleinen Ausstieg aus dem System zu praktizieren:
ABB. 1

\section{Gesellschaftskritik-Cluster nach Statusgruppen}

Angaben in Prozent

- Affirmative $\quad$ Korporatisten $\quad$ Individualisten Kritiker

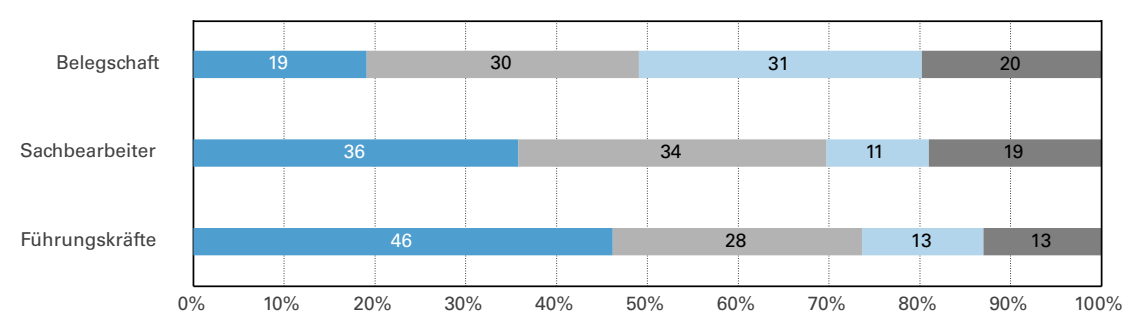

Quelle: Berechnung und Darstellung der Autoren.

„Und dann [...] sitz ich irgendwo ein paar Minuten bei mir daheim, das geht mir durch den Kopf und dann stell ich eigentlich für mich fest, wie gut dass es mir eigentlich geht und dass ich mich da, wo ich bin, eigentlich (...) ziemlich wohlfühl'. Und mir geht's halt prima, wenn ich mit meiner Familie wieder so wie jetzt vier Tage mal geschwind ein langes Wochenende ins Allgäu fahren kann auf unsere, auf unseren kleinen idyllischen Bauernhof, und ich muss nicht irgendwo oder nicht mehr das Streben nach mehr und ich muss jetzt irgendwo hinfliegen unbedingt, weil da alle anderen waren, oder ich muss jetzt ins Kino gehen und mir unbedingt den neuen James Bond angucken, [...]“ (Interview).

Die Konsequenz aus der Kritik an einer Ausbeutungsordnung, die sich vor allem außerhalb jenes Unternehmens findet, in welchem man selbst beschäftigt ist, ist der individuelle Ausstieg auf Zeit. Der Rückzug auf den idyllischen Bauernhof im Allgäu und das dort mögliche ungestörte Familienleben enthält eine höchst praktische Kritik an der Steigerungslogik des kapitalistischen Wettkampfsystems, das auch beim Konsum - sei es das neueste Smartphone oder der aktuelle Blockbuster - permanent zum Mitmachen zwingt.

Auch solche Handlungsstrategien lassen sich nicht als bloße Konsequenz eines „falschen Bewusstseins“ klassifizieren. Für Lohnabhängige, die etwas zu verlieren haben, ist, darauf hat Terry Eagleton hingewiesen, „eine gewisse Apathie durchaus vernünftig“" (Eagleton 2011, S. 225). ${ }^{3}$ „Es widerstrebe Menschen außerordentlich, ihre Situation

3 „Solange ein Gesellschaftssystem seinen Bürgern ein mageres Auskommen einbringt, ist es nicht unvernünftig, dass sie an dem festhalten, was sie haben, statt waghalsig in eine ungewisse Zukunft zu springen. Es gibt keinen Anlass, über einen solchen Konservatismus zu spotten“ (ebd.). 
zu verändern, solange sie von dieser Situation noch etwas erwarten können“" (ebd., S. 224). Genau dies trifft auf einen Großteil der von uns befragten Arbeiter und Angestellten zu. Sie können, aller Kritik an Missständen und Negativentwicklungen zum Trotz, von ihrer Arbeits- und Lebenssituation noch etwas erwarten. Die Aussicht auf grundlegende gesellschaftliche Veränderungen erscheint ihnen demgegenüber als vage und außerordentlich risikoreich. Daher „entwichtigen " die persönlichen Erfahrungen im Privaten, in der Familie und den eigenen sozialen Netzwerken die durchaus vorhandene kritische Sicht auf die Gesellschaft. Der alles vereinnahmenden Logik des Wettbewerbs- und Finanzkapitalismus vermag man immerhin den eigenen Entwurf des guten Lebens entgegenzusetzen, der sich - auch aufgrund der überdurchschnittlichen Entlohnung, die eine Beschäftigung „beim Z." noch immer bietet - wenigstens teilweise verwirklichen lässt. Schon mit Blick auf die eigenen Kinder und deren Zukunft scheint dieser Entwurf vom guten Leben allerdings bedroht. Und genau daraus erwächst eine kritische Haltung gegenüber der Gesellschaft. Es geht gar nicht darum, eine gegnerische Klasse zu benennen, deren Reichtum „von unten“ angeeignet werden muss. Den Befragten fällt es schwer, für die ausbeutenden Klassen überhaupt eine Bezeichnung zu finden. Sie denken in anderen Kategorien.

Tiefster Grund für ihre kritische Haltung ist offenbar ein Prinzip des ,immer Mehr“, das die gesamte Gesellschaft prägt. Ihm selbst, so macht der oben zitierte Befragte deutlich, ist ein solches Denken fremd. Realisierte sich sein Traum vom Lottogewinn - ein Synonym für die Befreiung von materiellen Zwängen -, so würde er sein Leben nicht grundlegend verändern. Er fühlt sich jetzt wohl, in und mit einem Leben, so wie es ist. Dieses Leben möchte er vor einer als bedrohlich empfundenen Zukunft verteidigen, ein Wunsch, der eine konservierende Grundhaltung konstituiert. Dem Befragten gilt die „kleine Welt“ der Familie nicht nur als Rückzugsraum, sondern als sozialer Zusammenhang, der ein gutes Leben ermöglicht. Und die „kleine Welt“ des Werks, das sichere Beschäftigung und hohe Löhne bietet, wird trotz aller Kritik an Missständen und Verschlechterungen subjektiv zur Bestandsgarantie für jene Lebensqualität, die man selbst nicht mehr missen möchte.

\section{Arbeitsbewusstsein und Wachstumskritik}

Dieser konservierende Grundzug prägt die alltägliche Gesellschafts- und Kapitalismuskritik typenübergreifend. Er findet sich auch bei Wettbewerbskorporatisten und moderaten Kritikern. Kritik am Finanzmarkt-Kapitalismus, der häufig als „Verzerrung“ eines an sich funktionsfähigen Systems gedeutet wird, paart sich in diesen Gruppen mit dem Wunsch nach einer sozialen Korrektur, nach einer Rückkehr zur sozialen Marktwirtschaft. Es wäre aber falsch, wollte man den bewahrenden Grundzug der alltäglichen Gesellschafts- und Kapitalismuskritik umstandslos mit politischem Konservativismus identifizieren. Eine solche Verbindung kann sich als Wahlverwandtschaft einstellen, das muss aber nicht zwingend der Fall sein. Vielmehr schließt die Verteidigung des eigenen guten Lebens subjektiv eine Wachstums- und Wettbewerbskritik ein, deren Massivität uns überrascht hat. Wie unsere qualitativen Befunde belegen, ist die Kritik an einer Steigerungslogik des „Immer mehr und nie genug!" ebenfalls typenübergreifend verbreitet. Sie bezieht sich sowohl auf die Arbeitserfahrungen als auch auf andere gesellschaftliche Phänomene:

„Und [...] wenn ich dann die Zapfsäulen anschaue, wer kann sich das alles noch leisten in dem Zusammenhang. Und dann ist auch wirklich, der Kollaps, der Klimakollaps, gell, also, (pfff) mit den fossilen Brennstoffen und da wird vielleicht auch selbst der Hybrid-Motor oder der HybridAntrieb [...] nicht einmal die Zwischenlösung. Was nützt mir das, wenn der Sprit zehn Euro kostet und ich brauch nur vier Liter gegenüber vorher von acht Litern oder sieben Litern. Dann hab ich auch nix gewonnen, weil, das HybridFahrzeug kostet auch mehr Geld in der Anschaffung, gell [...] auch die Elektronik hat ihre Rohstoffe, die sie brauchen, die irgendwann zu Ende sind“ (Interview).

In diesem Fall speist sich die Gesellschafts- und Kapitalismuskritik unmittelbar aus einer Beobachtung und subjektiven Bearbeitung ökologischer Krisen. Etwas weniger pointiert und durchaus kontrovers aufeinander bezogen finden wir die ökologisch inspirierte Kritik aber in allen Clustern. Viele Statements belegen, dass die Wachstums-, Klima- und Ressourcenproblematik in der Alltagskommunikation der Belegschaft Thema ist. Man weiß relativ genau, wie die Kolleginnen und Kollegen hinsichtlich der ökologischen Frage denken. Das ist nicht weiter verwunderlich, denn der Zusammenhang zwischen Ressourcen, allen voran dem Erdöl, und der Zukunft der eigenen Branche ist sämtlichen Interviewpartnern bewusst. Bei den intensiv Befragten gibt es keinen, der die Problematik leugnen würde. Stattdessen stoßen wir auf Ausweichargumentationen getreu dem Motto „Deutschland allein kann die Welt nicht retten!" Doch solche argumentationsstrategischen Manöver sind nicht die Regel. Auch die Furcht vor Arbeitsplatzverlusten und Beschäftigungsunsicherheit infolge eines ökologischen Strukturwandels ist präsent, aber deutlich weniger ausgeprägt als wir angenommen hatten. Die Befragten sehen realistisch, dass die Beschäftigung in der Branche angesichts der weltweiten Überkapazitäten zumindest im Inland nicht mehr wachsen wird. Zugleich vertrauen sie jedoch auf die eigene, vergleichsweise hohe Qualifikation und sind einigermaßen sicher, auch im Falle einer Umstellung auf Elektro-Autos oder einer noch weitergehenden Produktkonversion gebraucht zu werden. Aus dieser Perspektive findet ein sozialökologischer Umbau selbst bei den moderaten Gesellschaftskritikern Unterstützung.

Wiederum gilt hier das "gute“, weil innovative Unternehmen als Schlüssel zur Lösung gesellschaftlicher Proble- 
me. Es geht nicht darum, eine ökologische Orientierung gegen den Konzern durchzusetzen; vielmehr soll das Unternehmen sein Gewicht in die Waagschale werfen, um eine ökologische Transformation der Produkte und Produktionsweise in Gang zu setzen. Entscheidend für unsere Frage nach den Beweggründen für Kapitalismuskritik ist jedoch ein anderer Befund. Die ökologische Krise gilt vielen Befragten als Konsequenz jenes „Immer mehr und nie genug!“, das sie aus dem Betrieb, aber auch aus anderen Lebensbereichen kennen. Als Treiber wird ein verselbstständigtes Wettbewerbsprinzip identifiziert, dessen zerstörerische Wirkung in unterschiedlichen Kontexten erlebt wird. Dieses expansive Wettbewerbsprinzip ist aus dem betrieblichen Alltag bekannt und wird in zahlreichen Variationen beschrieben und kritisiert:

„[...] ich drück's mal so aus, wo ich angefangen hab in der Produktion haben wir ungefähr mit der gleichen Mitarbeiterzahl 290.000 Teile gebaut im Jahr. Das hat für mich bedeutet, für die Innenteile, wo ich arbeite, mit den älteren Maschinen eine Stückzahl von zwei- bis dreihundert Teilen am Tag. Und mittlerweile machen wir 1,2 Millionen Teile mit den gleichen Mitarbeitern“ (Interview).

Die einschneidende Erfahrung ist jedoch, dass dieser enorme Rationalisierungssprung nicht ausreicht. Schon legt der Konzern das nächste Rationalisierungsprogramm auf, schon geht es wieder darum, die Wettbewerbsposition des Unternehmens zu verbessern - und wieder bedeutet das, noch schneller, noch effizienter arbeiten zu müssen. Allerdings macht sich die Unersättlichkeit des Wettbewerbs nicht allein in Betrieb und Arbeitswelt bemerkbar; mehr und mehr durchdringt sie alle Poren der Gesellschaft und wirkt selbst auf die Lebenswelt von Kindern und Heranwachsenden ein. Diese Beobachtung belastet den zitierten Facharbeiter noch stärker als der betriebliche Rationalisierungsdruck.

Die Gesellschaft, das klingt in vielen Sequenzen an, ist für die Befragten zu einer Ansammlung von Wettkämpfen degeneriert, die das soziale Verhalten von Kindesbeinen an beeinflussen. Dieses Wettbewerbsprinzip ist unersättlich. Es erzeugt permanent Gewinner und Verlierer. Es wirkt in mehr oder minder allen gesellschaftlichen Erfahrungsräumen, und es ist dieses Wettkampfprinzip, das die Lebensqualität in der Wahrnehmung der Befragten teilweise bis zur Unerträglichkeit einschränkt. Die Kritik am Wettkampf aus Prinzip stellt offenbar eine Brücke zwischen individuellen Erfahrungen und den subjektiven Gesellschaftsbildern her. Und diese kognitive Brücke vermittelt auch zwischen den Erfahrungen in der „kleinen Welt“ des Betriebs, der privaten Welt der Familie und den Haltungen gegenüber der „großen Gesellschaftswelt“. Bei dieser Verbindung handelt es sich allerdings nur um lose Koppelungen, und es ist keineswegs klar, ob die Arbeitserfahrungen in Gesellschaftskritik münden oder ob umgekehrt eine kritische Gesellschaftssicht auch zu einer kritischen Deutung der Arbeitssituation führt. Die Einschätzung der gesellschaftlichen Missverhältnisse kann, muss aber nicht mit den betriebli- chen Erfahrungen übereinstimmen. Es besteht keine prinzipielle Kongruenz, zumal die Wirkmächtigkeit der eigenen Handlungsmöglichkeiten in den Augen vieler Befragter deutlich differiert: Den Arbeitsplatz glaubt man durch eigene Leistung erhalten zu können, der Einfluss auf gesellschaftliche Prozesse ist hingegen in der Selbstwahrnehmung gering.

\section{Schlussbemerkung}

Dass sich hinter dem Unbehagen am Finanzmarkt-Kapitalismus, das viele Befragte artikulieren, auch eine ökologisch inspirierte Wachstumskritik verbirgt, ist eine empirische Fundsache, von der wir aufgrund der begrenzten Reichweite des empirischen Materials nicht wissen, in welchem Maße sie verallgemeinert werden kann. Die arbeitspolitische Bedeutung dieser Fundsache lässt sich jedoch anhand einer Anekdote illustrieren. Als wir im Anschluss an eine Erhebung mit Betriebsräten zu Abend aßen, kam die Sprache auf die zeitgenössische Wachstumskritik. Eine unserer Gesprächspartnerinnen gab dieser ökologisch inspirierten Kritikvariante mit Blick auf ihre eigene Belegschaft keine Chance. Ohne Wachstum im Konzern, so ihr „kategorischer Imperativ“, sei Beschäftigungssicherung nicht möglich, und ohne Beschäftigungssicherung sei Interessenpolitik nicht denkbar. Wenn unsere Befunde auch nur annäherungsweise zutreffen, so eilt die alltägliche Kapitalismus- und Wachstumskritik eines Teils der Belegschaft ihren potenziellen betrieblichen und gewerkschaftlichen Repräsentanten offenbar voraus. Wachstumskritik ist nicht nur eine Orientierung, die sich typenübergreifend findet, das Wissen um die Ressourcenproblematik und den Klimawandel ist auch geeignet, den ansonsten zentralen Anspruch auf Beschäftigungssicherheit im Betrieb zu relativieren. Wenn überhaupt, so wären es ökologische Erfordernisse, die zumindest einen Teil der Befragten dazu motivieren würden, sich umzustellen und Beschäftigungssicherheit vor Ort gegen Produktkonversion und eine Ökologisierung industrieller Produktion einzutauschen.

Sicher, das müssen wir hinzufügen, haben solche Orientierungen nichts mit jenem Neokonservatismus gemein, der mit Wachstumskritik vor allem Wohlstandsverzicht ohne Umverteilung assoziiert (Miegel 2010, S. 162). Sperrig verhalten sich diese Bewusstseinsformen auch gegenüber einer Kritik, wie sie in Teilen der Degrowth-Bewegungen vorherrschend ist. Die Behauptung, „Neoliberale und Marxisten“ legitimierten bei ihrem Streit um die „gerechte Verteilung eines mutmaßlichen Ertrags menschlicher Leistung“ nur „die Inanspruchnahme einer Beute, die aus ökologischer Sicht erstens gar nicht hätte entstehen dürfen und die zweitens alles andere als ,verdient' oder ,erarbeitet “" worden sei (Paech 2013, S. 38), würde unsere Befragten, sollten sie mit ihr konfrontiert werden, geradewegs ins Lager der ökologi- 
schen Konterrevolution treiben. Das nicht etwa, weil es sich bei den Arbeitern und Angestellten um Neoliberale oder um Marxisten handelte. Sie wären von der Ignoranz einer politischen Philosophie abgeschreckt, die das Streben nach Verteilungsgerechtigkeit umstandslos als Triebkraft eines ökologisch zerstörerischen Konsumerismus brandmarkt. Auch in diesem Fall sind die befragten Arbeiter und Angestellten ihren intellektuellen Kritikern offenbar einen Schritt voraus. Selbstgenügsamkeit und Maßhalten ist für ihre Entwürfe eines sinnerfüllten Lebens durchaus ein Orientierungspunkt. Ihr praktischer Alltagsverstand sagt ihnen jedoch, dass eine wirkliche Transformation ohne den Umbau des gesamten Produktionsapparates und in einer von zunehmender Ungleichheit geprägten Gesellschaft nicht zu haben ist. Leitbilder einer ökologischen Transformation hätten sich stattdessen die Identifikation von Beschäftigten mit Betrieb, Werk, Region und Unternehmen aus einer Aneignungsperspektive (Sauer/Nies 2012, S. 58) heraus zunutze zu machen. Denn es ist keineswegs so, dass die Arbeitsstätte den Lohnabhängigen per se als defizitärer Ort erscheint, den es an die demokratischen Standards der Gesellschaft anzunähern gilt. Zumindest in einem Unternehmen mit starkem Betriebsrat und einflussreichen Gewerkschaften hält man die Chance, im eigenen Interesse erfolgreich intervenieren zu können, teilweise noch für deutlich größer als in anderen sozialen Feldern. Arbeitspolitisch käme es daher darauf an, in einer Weise an das „Wir sind

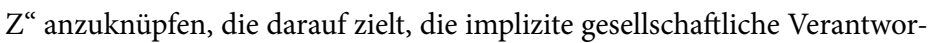
tung des Unternehmens offensiv einzuklagen und auszubauen. Dass dies gegenwärtig kaum geschieht, ist ein wichtiger Grund für die praktische Schwäche einer Wachstums- und Kapitalismuskritik, die im Alltagsbewusstsein von Arbeitern und Angestellten dennoch ständig präsent ist.

\section{LITERATUR}

Bahl, F./Staab, P. (2010): Das Dienstleistungsproletariat. Theorie auf kaltem Entzug, in: Mittelweg 36, 19 (6), S. 66-93

Beaud, S./Pialoux, M. (2004): Die verlorene Zukunft der Arbeiter, Konstanz Behr, M./Happ, A./Dörre, K./Elsner, M. (2013): Arbeitsbewusstsein und Interessenorientierung in einem ostdeutschen Unternehmen der optischen Industrie, in: Dörre, K./Happ, A./Matuschek, I. (Hrsg.), a.a.O., S. 54-84

Detje, R./Menz, W./Nies, S./Sauer, D. (2011): Krise ohne Konflikt? Interessenund Handlungsorientierungen im Betrieb - die Sicht von Betroffenen, Hamburg Dörre, K. (2014): Public Sociology - ein Konzept für die Arbeitsforschung, in: Wetzel, D./Hofmann, J./Urban, H.-J. (Hrsg.): Industriearbeit und Arbeitspolitik. Kooperationsfelder von Wissenschaft und Gewerkschaften, Hamburg,

S. $85-98$

Dörre, K./Hänel, A./Holst, H./ Matuschek, I. (2011): Guter Betrieb, schlechte Gesellschaft? Arbeits- und Gesellschaftsbewusstsein im Prozess kapitalistischer Landnahme, in: Koppetsch, C. (Hrsg.): Nachrichten aus den Innenwelten des Kapitalismus. ZurTransformation moderner Subjektivität, Wiesbaden,

S. $21-50$

Dörre, K./Happ, A./Matuschek, I. (Hrsg.) (2013): Das Gesellschaftsbild der LohnarbeiterInnen. Soziologische Untersuchungen in ost- und westdeutschen Industriebetrieben, Hamburg

Dörre, K./Matuschek, I. (2013): Kapitalistische Landnahmen, ihre Subjekte und das Gesellschaftsbild der LohnarbeiterInnen, in: Dörre, K./Happ, A./Matuschek, I. (Hrsg.), a.a.O., S. 29-53

Dubet, F. (2008): Ungerechtigkeiten. Zum subjektiven Empfinden am Arbeitsplatz, Hamburg

Eagleton, T. (2011): Warum Marx recht hat, Berlin

Institut für Demoskopie Allensbach (2012): Das Unbehagen am Kapitalismus. Eine Dokumentation des Beitrags von Prof. Dr. Renate Köcher in der FAZ Nr. 45 vom 22. Februar

Miegel, M. (2010): Exit. Wohlstand ohne Wachstum, Berlin

Neugebauer, G. (2007): Politische Milieus in Deutschland. Die Studie der

Friedrich-Ebert-Stiftung, Bonn
Paech, N. (2013): Befreiung vom Überfluss. Auf dem Weg in die Postwachstumsökonomie, München

Popitz, H./Bahrdt, H. P./Jüres, E. A./Kesting, H. (1957): Das Gesellschaftsbild des Arbeiters. Soziologische Untersuchungen in der Hüttenindustrie, Tübingen Sauer, D./Nies, S. (2012): Arbeit - mehr als Beschäftigung? Zur arbeitssoziologischen Kapitalismuskritik, in: Dörre, K./Sauer, D./Wittke, V. (Hrsg.): Kapitalismustheorie und Arbeit - Neue Ansätze soziologischer Kritik, Frankfurt a. M./ New York, S. 34-62

Schumann, M. (2013): Das Jahrhundert der Industriearbeit. Soziologische Erkenntnisse und Ausblicke, Weinheim/Basel

\section{AUTOREN}

KLAUS DÖRRE, Dr. habil., ist Professor für Arbeits-, Industrie-und Wirtschaftssoziologie und Ko-Direktor der DFG-Kollegforschergruppe „Postwachstumsgesellschaften“ an der Friedrich-Schiller-Universität Jena. Arbeitsschwerpunkte: Prekarität, Theorie kapitalistischer Landnahmen.

klaus.doerre@uni-jena.de

HAJO HOLST, PD. Dr., ist Wissenschaftlicher Mitarbeiter am Institut für Soziologie der Friedrich-Schiller-Universität Jena. Arbeitsschwerpunkte: Corporate Governance, Finanzialisierung, Flexibilisierung und Prekarisierung von Arbeit, industrielle Beziehungen.

hajo.holst@uni-jena.de

INGO MATUSCHEK, Dr. rer. soc., ist Lehrbeauftragter an der Friedrich-SchillerUniversität Jena. Arbeitsschwerpunkte: Flexibilisierte und subjektivierte Arbeit, qualitative Methoden, Soziologie politischen Handelns.

ingo.matuschek@uni-jena.de 